\title{
Effect of tricuspid regurgitation and right ventricular dysfunction on long-term mortality in patients undergoing cardiac devices implantation: >10-year follow-up study
}

Nikolaos Papageorgiou ${ }^{1,2}$, Debbie Falconer ${ }^{3}$, Nikolas Wyeth ${ }^{3}$, Guy Lloyd ${ }^{1,3,4,5}$, Denis Pellerin ${ }^{1}$,

Elsya Speechly-Dick ${ }^{3,4}$, Martin Lowe ${ }^{2}$, Edward Rowland ${ }^{2}$, Pier D. Lambiase ${ }^{2,4}$, Anthony W. Chow ${ }^{2,}$ Sanjeev Bhattacharyya ${ }^{1,3,4,5}$

${ }^{1}$ Echocardiography Laboratory, Barts Heart Centre, St Bartholomew's Hospital, London, UK

${ }^{2}$ Electrophysiology Department, Barts Heart Centre, St. Bartholomew's Hospital, London, UK

${ }^{3}$ University College London Hospitals NHS Trust, London, UK

${ }^{4}$ Institute of Cardiovascular Sciences, UCL, London, UK

${ }^{5}$ William Harvey Research Institute, Queen Mary University of London, London, UK

Running title:

Word count:

\section{Corresponding author:}

Dr Sanjeev Bhattacharyya MD MRCP FESC

Barts Heart Centre, St. Bartholomew's Hospital

West Smithfield, EC1A 7BE

London, United Kingdom

Email: Sanjeev.Bhattacharyya@ bartshealth.nhs.uk 


\begin{abstract}
:
Background: The long-term effect of tricuspid regurgitation (TR) has on and long-term mortality. In the present study, we sought to examine whether patients undergoing an implantable cardiac device procedure (pacemaker-PPM, implantable cardiac defibrillator-ICD or cardiac resynchronisation therapy pacemaker/defibrillator-CRT-D/P) have an increased risk of TR and to determine the effect of this on long-term survival.
\end{abstract}

Methods: A total of 326 patients who underwent device implant and had pre- and post-implant transthoracic echocardiogram were included in the analysis. New heart failure (HF) onset and allcause mortality were the study endpoints over a follow-up period of median 11.8 years.

Results: Pre-implant, none/trivial, moderate and severe TR were present in 304 (93.3\%), 12 $(3.7 \%)$ and $10(3.1 \%)$ patients, respectively. TR grade increased post implant in $150(46 \%)$ patients. There was a significant increase in the proportion of patients with RV dysfunction after implantation (89 (27.3\%)) compared to pre-implantation $(44(13.5 \%)), \mathrm{p}<0.0001$. Moderate or greater TR was an independent predictor of new HF onset [OR:4.50 (95\%CI: 2.06-9.81), $\mathrm{p}=0.0002]$. Independent predictors of mortality were post-pacemaker $\geq$ moderate TR $[\mathrm{HR}: 4.18$ (95\%CI: $2.67-6.55) \mathrm{p}<0.0001]$ and RV impairment [HR: 2.15 (95\% CI: $1.30-3.56) \mathrm{p}=0.01)$.

Conclusions: Worsening TR and RV dysfunction post device implantation is common. Postimplant moderate or greater TR is associated with adverse RV function and increased risk of new onset HF and poor long term (>10 years) survival. 
Keywords: tricuspid regurgitation, right ventricular function, pacemaker, implantable cardiac defibrillator 


\section{Introduction}

There has been steady growth in the rate of implantation of permanent pacemakers over the past few decades (1). Implantation of transvenous cardiac devices including permanent pacemakers (PPM), implantable cardiac defibrillators (ICD) and cardiac resynchronization therapy devices (CRT-D/P) can result in significant tricuspid regurgitation (TR) either due to damage/disruption of the tricuspid valve or asynchrony, resulting from abnormal RV activation $(2,3)$.

Although early studies suggested no change in TR after devices implant (4), the vast majority demonstrated a significant association between device implant and TR with a significant increase in the severity of TR from pre- to post-permanent lead implantation $(5,6)$. In patients undergoing ICD or PPM insertion it was found that the prevalence of moderate/severe TR increased from $27 \%$ to $31 \%$ by 1 month and to $35 \%$ at 4 years post implantation (7).

Further studies have attempted to assess the role of TR on the mortality of patients undergoing device implant. Delling et al, showed an increased risk of mortality over a median follow-up period of 0.7 years (8). Similarly, in a retrospective cohort of 239 patients, significant lead-induced TR was associated with worse long-term survival in a median of just under 5 years (9).

Transvenous devices are implanted for the lifetime of a patient. At present, it is uncertain what the long-term effect of TR has on right ventricular (RV) remodelling, heart failure and longterm mortality. Therefore, in the present study, we sought to examine whether patients undergoing cardiac device implantation (PPM, ICD or CRT-D/P) have an increased risk of TR and to determine the effect of this on long-term (>10 years) survival. 


\section{Methods}

\section{Study population}

Three-hundred and sixty-five patients who underwent cardiac device implantation at University College London Hospitals NHS Trust, London, United Kingdom between 2004 and 2007 and had a pre- and post-implant transthoracic echocardiogram (TTE) were identified. The pre-implant TTE was required to $<1$ year prior to the date of implant. Thirty-nine patients were excluded from the study due to incomplete data and therefore, a total of 326 patients were included in our retrospective analysis.

Patient demographic/clinical characteristics were extracted from the electronic records. These included age, gender, cardiac risk factors including diabetes, hypertension, hyperlipidemia, smoking status, coronary artery disease (Table 1). We excluded patients with temporary pacing leads or those without pre- or post-procedure. The study was approved by Health Research Authority United Kingdom (awaiting approval then add reference number).

\section{Echocardiographic data}

All study participants underwent comprehensive two-dimensional transthoracic echocardiogram with a commercially available system. Left ventricular (LV) function was assessed using Simpson's biplane method or visual estimate. Normal, Mildly Impaired, Moderately Impaired, Severely Impaired corresponded to an ejection fraction of $\geq 55 \%, 45-54 \%, 30-44 \%,<30 \%$, respectively. Right ventricular size was measured using the four chamber view in diastole at the tricuspid annulus level. Right ventricular function was measured using tricuspid annular plane excursion (TAPSE) and RV systolic velocities (RV S). Impaired RV function was defined as either a TAPSE $<1.5 \mathrm{~cm}$ or RV $\mathrm{S}<10 \mathrm{~cm} / \mathrm{s}$. Tricuspid regurgitation was graded as none/trivial, mild, 
moderate or severe based on an integrated approach using regurgitant jet area, vena contracta and proximal isovelocity surface area and continuous wave Doppler profiles. Pulmonary artery systolic pressure was estimated using the tricuspid continuous wave Doppler signal and inferior vena cava size and collapsibility.

\section{Follow-up period and endpoint}

Follow-up was calculated from the time of their pacemaker implant to the last clinic follow up or documented event (median 11.8 years). New heart failure (HF) onset and all-cause mortality were identified from the hospital electronic patient record. This links to the national health service spine which records all patient death in the United Kingdom.

\section{Statistical analysis}

Descriptive data are presented as numbers and percentages or median and inter-quartile range. The Chi-square test was used for comparing ratios and categorical variables. Kaplan-Meier curves were used to illustrate the association of different severity of TR and RV function with the study endpoints. The log rank test was used to assess for the presence of differences. Logistic regression was used to identify predictors of new-onset HF post-pacemaker implantation. Cox regression was used in the analysis of time-to-event data to identify predictors of mortality. Results with $P<0.05$ were regarded as significant. PASW Statistics (SPSS Inc, Chicago, IL) version 18.0 was used for statistical analysis.

\section{Results}

Baseline characteristics of the 326 included in our study are presented in table 1. One hundred and seven (32.8\%) patients had RV pacing leads and 219 (67.2\%) patients had RV defibrillator leads. 


\section{Tricuspid regurgitation}

At baseline, $n$ trivial, mild, moderate and severe TR were present in 248 (76.1\%), 56 (17.2\%), 12 (3.7\%) and $10(3.1 \%)$ patients, respectively. The degree of TR increased in $150(46 \%)$ patients. There was an increase of one grade of TR in 106/150 (70.6\%) patients, two grades in 35/150 $(23.3 \%)$ patients and three grades in $9 / 150(6 \%)$ patients. There was no change of grade in 165 $(50.6 \%)$ patients. There was a reduction of grade in $11(3.4 \%)$ patients.

\section{Right ventricular dysfunction}

There was a significant increase in the proportion of patients with RV dysfunction after implantation $(89(27.3 \%))$ compared to pre-implantation $(44(13.5 \%)), \mathrm{p}<0.0001$. The proportion of patients with RV dysfunction increased as the grade of TR worsened. RV dysfunction was present in 18/129 (14\%) patients with no or trivial TR, 28/123 (22.8\%) patients with mild TR, 24/49 (49\%) patients with moderate TR and 19/25 (76\%) patients with severe TR.

\section{New Heart Failure onset}

There were 49/326 (15\%) patients who developed new onset HF post-pacemaker implantation. Moderate or more ( $\geq$ moderate) TR was present in $27 / 49(55 \%)$ of patients with new onset HF compared to $60 / 277(21.7 \%)$ of patients without new onset HF, p<0.0001. Moderate or more TR was an independent predictor of new HF (Table 2).

\section{All-cause mortality}

During a median follow-up of 11.8 years, the endpoint of death was reached in 105 patients. Independent predictors of mortality were post pacemaker $\geq$ moderate TR [HR: 4.18 (95\% CI 2.67 - 6.55) $\mathrm{p}<0.0001]$ and RV impairment [HR: 2.15 (95\% CI 1.30 - 3.56) p=0.01] (Table 3). In 
contrast, pre-procedure LV and RV function and TR were not predictors. Kaplan Meier curves (Figure 1) showed a significant reduction in survival of patients with $\geq$ moderate TR compared to those with <moderate TR ( $<<0.0001)$. Kaplan Meier curves (Figure 2) also showed a significant reduction in survival of patients with post-procedure RV dysfunction compared to those without RV dysfunction $(\mathrm{p}<0.001)$.

\section{Discussion}

This study, with more than 10 years follow-up, has shown that the degree of post-implant TR is associated with the development of RV dysfunction. Importantly, post-implant $\geq$ moderate TR was a predictor of new onset post-implant HF. Both post-implant $\geq$ moderate TR and RV dysfunction were independent predictors of long-term mortality.

An increase in the severity of TR from pre- to post-permanent lead implantation by 1 or 2 grades in patients is well recognised(5, 6). In a cohort of 1596 patients undergoing ICD or PPM insertion it was found that the prevalence of moderate/severe TR increased from $27 \%$ to $31 \%$ at 1 month and to $35 \%$ at 4 years (7). The mechanism of post-device pacemaker TR is varied. Possible aetiologies include leaflet impingement, adherence, perforation, interference with chordal apparatus and delayed right ventricular activation (10). Two-dimensional (2D) echocardiography can be used to grade TR as well as its effect on RV. However, ability of $2 \mathrm{D}$ techniques to delineate relationship between lead and valve leaflets is limited (11). Mediratta et al (9) showed threedimensional (3D) echocardiography could depict lead position in relation to tricuspid leaflets and identify aetiology of valve dysfunction in $90 \%$ of patients. Active RV pacing is associated with a significant increase in TR grade (12). 
Despite, studies examining, the prevalence of post-device TR, there remains a lack of consensus on the optimal management of these patients. Several studies have examined the short and mid-term outcomes of these patients. Delling et al (8) showed that among 169 patients with available pre- and post-implantation echocardiograms and less than 1 year follow-up, the presence of TR was associated with an increased risk of death. Furthermore, Höke U et al (9) showed in 239 device recipients with echocardiographic evaluation and nearly 5 years follow-up, significant leadinduced TR had worse long-term survival and/or more HF related events. However, patients with devices often have implants lifelong. Our study shows with $>10$ years follow-up $\geq$ moderate TR is associated with adverse RV dysfunction, increased risk of HFe and worse mortality.

In our study, the mortality difference between those with and without device-induced significant TR widens from implant to 5 and then 10 years. Given this prognostic data, we would recommend routine evaluation of TR and RV function post-implant. The clinical conundrum is given the poor outcome of post-plant TR, is whether lead revision/ extraction should be considered. At present, we have no data to base a decision on. Although, a clinical trial testing whether lead revision influence outcome may seem attractive there are several potential problems. Each patient will have individual circumstances including aetiology of device-induced TR, symptom status, indication for device and co-morbidities. In addition, despite 3D echocardiography improving diagnosis of pacemaker-related TR, there remains uncertainty whether lead removal will reduce the grade of TR. For example, lead adherence to the leaflet may lead to permanent damage which will not resolve after lead removal. In addition, the effect of TR on RV remodelling may lead to annular dilatation causing additional functional TR which may not improve post-lead revision. Therefore, any future studies need to be focussed on identifying specific patient groups who may benefit from lead manipulation. 
The main limitation of this study is the retrospective nature of analysis which may make the study susceptible to selection bias. However, this has allowed long term (>10 year) follow-up to be analysed. In addition, grading of TR is semi-quantitative using a range of parameters to decide on the overall grade. However, this is the current guideline recommended method for evaluation of TR. The cause of worsening grade of TR was not studied by 3D echocardiography and therefore the exact aetiology could not be determined.

\section{Conclusions}

Worsening TR commonly occurs after device implantation. Post-implant moderate or greater TR is associated with adverse RV function and increased risk of new onset HF and poor long term (>10 years) survival. 


\section{References}

1. Greenspon AJ, Patel JD, Lau E, Ochoa JA, Frisch DR, Ho RT, Pavri BB, Kurtz SM. Trends in permanent pacemaker implantation in the United States from 1993 to 2009: increasing complexity of patients and procedures. J Am Coll Cardiol. 2012 Oct 16;60(16):1540-5.

2. Marijon E, Trinquart L, Otmani A, et al., EVADEF Investigators. Predictors for short-term progressive heart failure death in New York Heart Association II patients implanted with a cardioverter defibrillator: the EVADEF study. Am Heart J 2010; 159:659-64.e1.

3. Packer DL, Prutkin JM, Hellkamp AS, et al. Impact of implantable cardioverterdefibrillator, amiodarone, and placebo on the mode of death in stable patients with heart failure: analysis from the Sudden Cardiac Death in Heart Failure trial. Circulation 2009;120:2170-6.

4. Leibowitz DW1, Rosenheck S, Pollak A, Geist M, Gilon D. Transvenous pacemaker leads do not worsen tricuspid regurgitation: a prospective echocardiographic study. Cardiology. 2000;93(1-2):74-7.

5. Klutstein M1, Balkin J, Butnaru A, Ilan M, Lahad A, Rosenmann D. Tricuspid incompetence following permanent pacemaker implantation. Pacing Clin Electrophysiol. 2009 Mar;32 Suppl 1:S135-7. doi: 10.1111/j.1540-8159.2008.02269.x.

6. Kim JB1, Spevack DM, Tunick PA, Bullinga JR, Kronzon I, Chinitz LA, Reynolds HR. The effect of transvenous pacemaker and implantable cardioverter defibrillator lead placement on tricuspid valve function: an observational study. J Am Soc Echocardiogr. 2008 Mar;21(3):284-7. Epub 2007 Jul 2.

7. Al-Bawardy R1, Krishnaswamy A, Rajeswaran J, Bhargava M, Wazni O, Wilkoff B, Tuzcu EM, Martin D, Thomas J, Blackstone E, Kapadia S. Tricuspid regurgitation and 
implantable devices. Pacing Clin Electrophysiol. 2015 Feb;38(2):259-66. doi: 10.1111/pace.12530. Epub 2014 Nov 7.

8. Delling FN1, Hassan ZK2, Piatkowski G3, Tsao CW4, Rajabali A4, Markson LJ5, Zimetbaum PJ4, Manning WJ6, Chang JD4, Mukamal KJ7. Tricuspid Regurgitation and Mortality in Patients With Transvenous Permanent Pacemaker Leads. Am J Cardiol. 2016 Mar 15;117(6):988-92. doi: 10.1016/j.amjcard.2015.12.038. Epub 2016 Jan 6.

9. Höke U1, Auger D2, Thijssen J2, Wolterbeek R3, van der Velde ET2, Holman ER2, Schalij MJ2, Bax JJ2, Delgado V2, Marsan NA2. Significant lead-induced tricuspid regurgitation is associated with poor prognosis at long-term follow-up. Heart. 2014 Jun;100(12):960-8. doi: 10.1136/heartjnl-2013-304673. Epub 2014 Jan 21.

10. Mediratta A, Addetia K, Yamat M, Moss JD, Nayak HM, Burke MC, Weinert L, Maffessanti F, Jeevanandam V, Mor-Avi V, Lang RM. 3D echocardiographic location of implantable device leads and mechanism of associated tricuspid regurgitation. JACC Cardiovasc Imaging. 2014 Apr;7(4):337-47.

11. Seo Y, Ishizu T, Nakajima H, Sekiguchi Y, Watanabe S, Aonuma K. Clinical utility of 3dimensional echocardiography in the evaluation of tricuspid regurgitation caused by pacemaker leads. Circ J 2008;72:1465-70.

12. Vaturi M1, Kusniec J, Shapira Y, Nevzorov R, Yedidya I, Weisenberg D, Monakier D, Strasberg B, Sagie A.Right ventricular pacing increases tricuspid regurgitation grade regardless of the mechanical interference to the valve by the electrode. Eur J Echocardiogr. 2010 Jul;11(6):550-3. doi: 10.1093/ejechocard/jeq018. Epub 2010 Feb 25. 
Table 1. Baseline Demographics

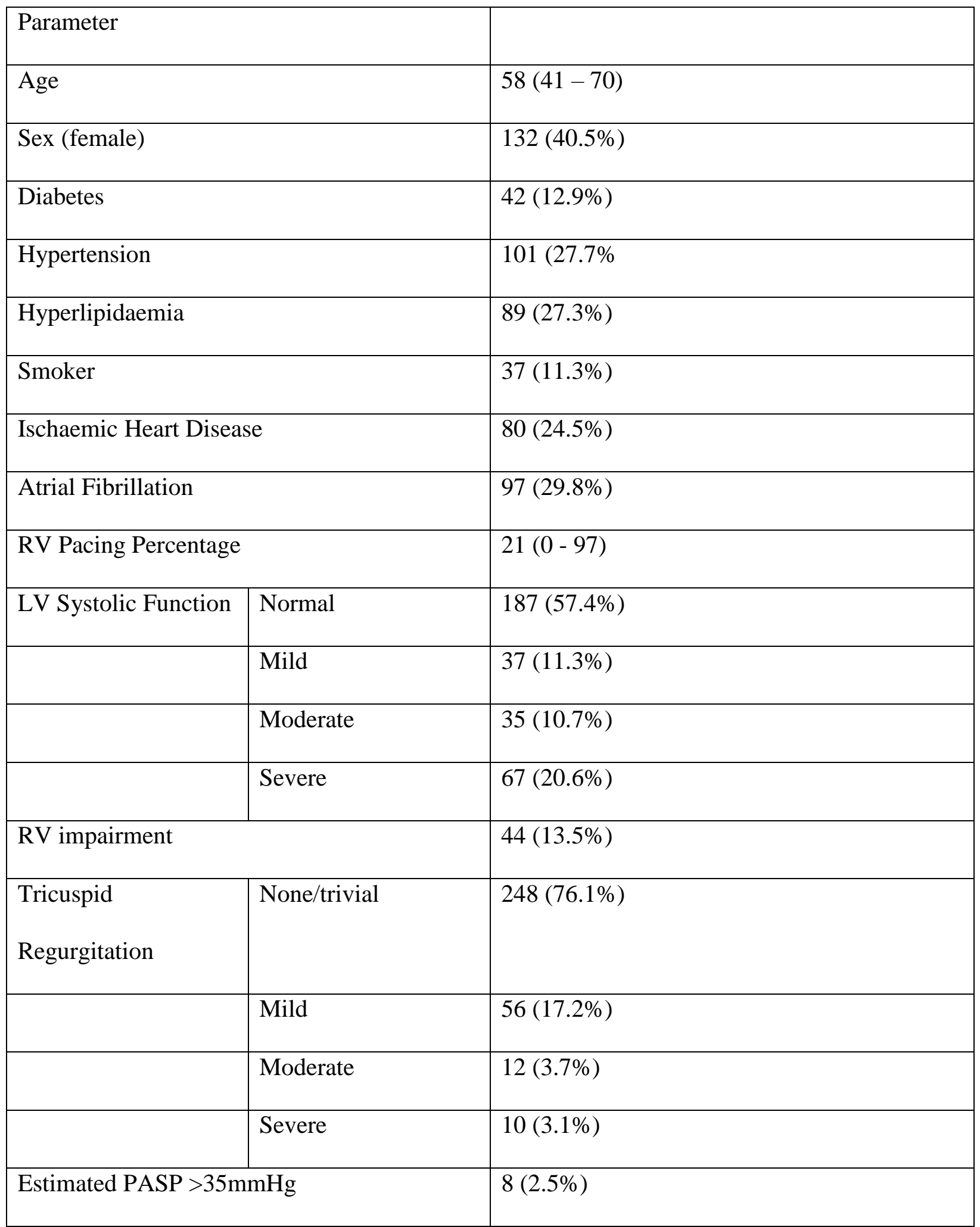

Left Ventricular (LV), Right Ventricular (RV), Pulmonary Artery Systolic Pressure (PASP) 
Table 2. Predictors of New-Onset Heart Failure Post Pacemaker

\begin{tabular}{|c|c|c|c|}
\hline Parameter & Odds ratio & Confidence Interval & $\mathrm{P}$ value \\
\hline Age & 1.01 & 0.9901 .03 & 0.30 \\
\hline Diabetes & 0.43 & $0.13-1.43$ & 0.17 \\
\hline Hypertension & 1.05 & $0.44-2.52$ & 0.92 \\
\hline Ischaemic Heart Disease & 1.25 & $0.52-3.02$ & 0.61 \\
\hline Atrial Fibrillation & 0.64 & $0.29-1.41$ & 0.27 \\
\hline Percentage RV Pacing & 1.00 & 0.9901 .01 & 0.65 \\
\hline $\begin{array}{l}\text { Pre-implant LV } \\
\text { impairment }\end{array}$ & 0.53 & $0.25-1.11$ & 0.09 \\
\hline $\begin{array}{l}\text { Pre-implant RV } \\
\text { impairment }\end{array}$ & 0.86 & $0.29-2.53$ & 0.78 \\
\hline Pre-implant $\geq$ Moderate TR & 0.49 & $0.14-1.75$ & 0.27 \\
\hline $\begin{array}{l}\text { Post implant } \geq \text { moderate } \\
\text { TR }\end{array}$ & 4.50 & $2.06-9.81$ & 0.0002 \\
\hline $\begin{array}{l}\text { Post-implant RV } \\
\text { impairment }\end{array}$ & 1.74 & $0.68-4.41$ & 0.24 \\
\hline
\end{tabular}

Left Ventricular (LV), Right Ventricular (RV), Tricuspid Regurgitation (TR) 
Table 3. Cox Regression Analysis For Mortality

\begin{tabular}{|c|c|c|c|}
\hline Parameter & Hazard Ratio & Confidence Interval & $\mathrm{P}$ value \\
\hline Age & 1.01 & $0.99-1.02$ & 0.28 \\
\hline Diabetes & 0.87 & $0.48-1.58$ & 0.64 \\
\hline Hypertension & 0.88 & $0.52-1.51$ & 0.65 \\
\hline $\begin{array}{l}\text { Ischaemic Heart } \\
\text { Disease }\end{array}$ & 1.24 & $0.72-2.12$ & 0.43 \\
\hline Atrial Fibrillation & 1.26 & $0.81-1.98$ & 0.31 \\
\hline $\begin{array}{l}\text { Percentage RV } \\
\text { Pacing }\end{array}$ & 1.00 & $1.00-1.01$ & 0.48 \\
\hline $\begin{array}{l}\text { Pre-implant LV } \\
\text { impairment }\end{array}$ & 0.73 & $0.47-1.16$ & 0.18 \\
\hline $\begin{array}{l}\text { Pre-implant RV } \\
\text { impairment }\end{array}$ & 0.67 & $0.35-1.31$ & 0.24 \\
\hline $\begin{array}{l}\text { Pre-implant } \\
\geq \text { Moderate TR }\end{array}$ & 0.45 & $0.19-1.06$ & 0.07 \\
\hline $\begin{array}{l}\text { Post implant } \\
\geq \text { moderate TR }\end{array}$ & 4.18 & $2.67-6.55$ & $<0.0001$ \\
\hline $\begin{array}{l}\text { Post-implant RV } \\
\text { impairment }\end{array}$ & 2.15 & $1.30-3.56$ & 0.01 \\
\hline
\end{tabular}

Left Ventricular (LV), Right Ventricular (RV), Tricuspid Regurgitation (TR) 
Figure Legends

Figure 1. Kaplan-Meier Curves demonstrating survival differences between patients with $\geq$ moderate tricuspid regurgitation compared to patients with $<$ moderate tricuspid regurgitation.

Figure 2. Kaplan-Meier curves showing survival differences between patients with and without right ventricular dysfunction. 